\title{
AUTOPERCEPÇÃO DOS PACIENTES FIBROMIÁLGICOS SOBRE O TRATAMENTO FISIOTERÁPICO
}

\author{
Self-perception of patients with fibromyalgia on the \\ physiotherapy treatment
}

\author{
Autopercepción de los pacientes con fibromialgia sobre el \\ tratamiento fisioterapico
}

\section{RESUMO}

Objetivo: Compreender a autopercepção do paciente com fibromialgia em relação à sua patologia e à fisioterapia e mostrá-la como um instrumento de aperfeiçoamento na prática dos profissionais de saúde Métodos: Pesquisa com abordagem qualitativa, com características descritivas e exploratórias, realizada em um centro de tratamento fisioterápico do município de Fortaleza-Ceará, em 2013. Fizeram parte do estudo 10 pessoas com diagnóstico clínico de fibromialgia em tratamento fisioterapêutico. Os dados foram coletados através de entrevista semiestruturada e analisados à luz da análise de conteúdo. Pôde-se agrupar o texto com os seguintes temas: Relação entre dor e cansaço; A fibromialgia resumida como uma dor, pessoal, física e mental; Fisioterapia, a oficina funcional do corpo; A perspectiva do sadio e a incerteza; e O fisioterapeuta humanizado. Resultados: A dor foi relatada como o maior fator incapacitante e por isso se dizem cansados e estressados. Compreendem a importância da Fisioterapia no seu processo de adaptação de limites físicos e na convivência com a dor, e conhecem o fisioterapeuta como um profissional humanizado. Conclusão: Os relatos mostram que os pacientes, em atendimento fisioterapêutico, têm a percepção de sua condição como enfermo, sabendo da importância da fisioterapia na sua condição funcional. As falas dão um feedback, ao profissional, que identifica os objetivos e os fatores que interferem no tratamento, podendo sugerir também a necessidade de intervenções de outras especialidades.

Descritores: Narração; Fibromialgia; Fisioterapia.

\section{ABSTRACT}

Objective: To understand the self-perception of the patient with fibromyalgia regarding the pathology and physiotherapy and show it as an instrument for improving the practice of health professionals. Methods: A qualitative, exploratory and descriptive research conducted at a physiotherapy center in the municipality of Fortaleza, Ceará, in 2013. The study comprised 10 people with a clinical diagnosis of fibromyalgia undergoing physiotherapy treatment. Data were collected through semi-structured interview and underwent content analysis. The text could be grouped into the following themes: Relationship between pain and fatigue; Fibromyalgia defined as a personal, physical and mental pain; Physiotherapy, the functional workshop of the body; The idea of being healthy and uncertainty; and the humanized physiotherapist. Results: Pain was reported as the most disabling factor and, because of that, they feel tired and stressed. They understand the importance of physiotherapy in the process of adaptation to physical limits and coping with pain, and recognize the physiotherapist as a humanized professional. Conclusion: The reports show that patients undergoing physiotherapy recognize they are sick and know the importance of physiotherapy for their functional condition. The speeches give a feedback to the professional who identifies the objectives and factors that interfere with treatment, and may also suggest the need for interventions by other professionals.

Descriptors: Narration; Fibromyalgia; Physical Therapy Specialty.
Artigo Original
1) Universidade de Fortaleza - UNIFOR Fortaleza (CE) - Brasil

2) Fisioterapeuta pela Universidade de Fortaleza - UNIFOR - Fortaleza (CE) -

Brasil

3) Núcleo de Atendimento Médico Integrado - NAMI - Universidade de Fortaleza - UNIFOR - Fortaleza (CE) Brasil

4) Hospital Geral Dr. Waldemar Alcântara HGWA - Fortaleza (CE) - Brasil
Recebido em: 23/03/2014

Revisado em: 22/04/2014 Aceito em: 18/05/2014 


\section{RESUMEN}

Objetivo: Comprender la autopercepción del paciente con fibromialgia respecto su patología y la fisioterapia y presentarla como instrumento de perfeccionamiento en la práctica de los profesionales de la salud. Métodos: Investigación de abordaje cualitativo con características descriptivas y exploratorias realizada en un centro de tratamiento fisioterápico del municipio de Fortaleza-Ceará, en 2013. Participaron del estudio 10 personas con diagnóstico clínico de fibromialgia y en tratamiento de fisioterapia. Los datos fueron recogidos a través de entrevista semi-estructurada y analizados a la luz del análisis de contenido. Se agrupo el texto en los siguientes temas: Relación de dolor y cansancio; La fibromialgia resumida como un dolor personal, fisico y mental; Fisioterapia, el taller funcional del cuerpo; La perspectiva del sano y la incertidumbre; y El fisioterapeuta humanizado. Resultados: El dolor fue relatado como el mayor factor incapacitante y por ello se dicen cansados y estresados. Los pacientes comprenden la importancia de la Fisioterapia en su proceso de adaptación de límites físicos y vivencia con el dolor $y$ reconocen el fisioterapeuta como un profesional humanizado. Conclusión: Los relatos muestran que los pacientes asistidos por la fisioterapia tienen la percepción de su condición de enfermo consciente de la importancia de la Fisioterapia en su condición funcional. Las hablas ofrecen un feedback al profesional que identifica los objetivos y los factores que interfieren en el tratamiento lo que también sugiere la necesidad de intervenciones de otras especialidades

Descriptores: Narración; Fibromialgia; Fisioterapia.

\section{INTRODUÇÃO}

A fibromialgia é classificada como uma doença do sistema muscular esquelético associada a distúrbios mentais somatoformes, sendo que na França utiliza-se o termo de "síndrome de polialgia difusa idiopática"(1) para classificá-la.

Essa doença é um distúrbio crônico. Geralmente, a primeira consulta ao médico se dá vários meses ou anos após o início dos sintomas, os quais permanecem regularmente estáveis pelos anos seguintes, sendo a dor muscular difusa seu principal sintoma, comumente presente em mulheres entre 30 e 50 anos de idade. Considera-se que ansiedade e distúrbios depressivos, frequentemente observados em pacientes com fibromialgia, são as causas basais da síndrome, ou são vistos como sintomas da doença. Uma associação com hipocondria neurótica e histeria também já foi postulada ${ }^{(2)}$.

$\mathrm{O}$ tratamento de pacientes com essa patologia requer abordagem multidisciplinar, sempre considerando a opinião do paciente. Pode-se associar tratamento não farmacológico e farmacológico, melhorando a qualidade de vida com suporte psicológico, médico, social e ocupacional ${ }^{(2)}$.

Assim, no tratamento fisioterápico, deve-se ter como objetivo a restauração da função e de estilos de vida, promovendo a saúde, o bem-estar e a qualidade de vida dos pacientes, sendo integrante do trabalho multidisciplinar e educativo, no qual os profissionais de saúde se proponham a informar e instruir corretamente, como no caso da necessidade de outras intervenções. Dessa forma, é importante o profissional de fisioterapia saber quando e como direcionar o paciente para outros tipos de acompanhamento, como, por exemplo, o psicológico. Uma das formas é a atenção na história dos pacientes, visto que atua como modelo geral de compreensão dos fenômenos psicossociais ${ }^{(3)}$. Para isto, o profissional da saúde precisa estar atento à escuta dessas narrativas, pois elas fazem uma reconstrução da identidade para que a aplicação de meios terapêuticos produza o efeito esperado, fazendo o enfermo se sentir sujeito dos processos e assuma com autonomia as mudanças necessárias em sua vida ${ }^{(4)}$.

O paciente diagnosticado com fibromialgia mostrase um indivíduo bastante presente nos ambulatórios de fisioterapia, porém com obstáculos durante o tratamento por sua complexidade devido seu difícil diagnóstico, a cronicidade dos múltiplos sintomas e o prognóstico sem cura estabelecida. Diante das necessidades vivenciadas pelos autores do presente estudo, profissionais da fisioterapia, despertou-se o interesse em observar o que estes pacientes tinham a relatar sobre o tratamento.

Assim, objetivou-se compreender a autopercepção do paciente com fibromialgia em relação a sua patologia e a fisioterapia, e mostrá-la como um instrumento de aperfeiçoamento na prática dos profissionais de saúde.

\section{MÉTODOS}

A presente pesquisa, de abordagem qualitativa, com características descritivas e exploratórias, ocorreu em um centro de tratamento fisioterápico de uma unidade básica de saúde do município de Fortaleza, Ceará, pertencente à Regional VI, no período de agosto a setembro de 2013.

$\mathrm{Na}$ referida instituição existe um programa multidisciplinar composto por fisioterapeutas, médico reumatologista, farmacêuticos, terapeutas ocupacionais e psicólogos, que acompanham um grupo de 30 pacientes diagnosticados com fibromialgia, desde 2009. O paciente fibromiálgico, além de receber orientações, prescrições de medicamentos e acompanhamento psicológico, recebe as seguintes propostas de tratamento fisioterápico: auto-alongamentos, hidroterapia, pilates e atendimento ambulatorial convencional. 
Incluíram-se na pesquisa pessoas com diagnóstico clínico de fibromialgia em tratamento fisioterapêutico há mais de 6 meses e maiores de 18 anos. Não participaram do estudo os pacientes com algum distúrbio cognitivo e de fala que pudesse inviabilizar a entrevista. Para selecionar a amostra, escolheram-se 10 pacientes entre os 30 inscritos na instituição, indicados pelos profissionais da instituição, que através da convivência demonstraram boa oratória, discernimento e capacidade em desenvolver um raciocínio lógico nas formulações de respostas quando arguidos.

Para a coleta de dados utilizou-se uma entrevista semiestruturada, contendo duas partes. A primeira contemplou os dados de identificação dos entrevistados, com as seguintes variáveis: sexo, idade, escolaridade, atividade laboral; e a segunda parte continha as seguintes questões norteadoras, especialmente desenvolvidas para a presente pesquisa: a) Atualmente, como o(a) senhor(a) se define como uma pessoa com fibromialgia? b) Descreva como o(a) senhor(a) entende a fibromialgia? c) O que o(a) senhor(a) acha do tratamento de fisioterapia? d) Em relação à sua doença, quais suas expectativas, o que o(a) senhor(a) espera para o futuro? e) Como o(a) senhor(a) vê a postura e o conhecimento dos profissionais de fisioterapia em relação à sua doença?

A entrevista foi realizada por um único pesquisador treinado, em uma sala reservada, em ambiente confortável, na instituição em que os pacientes eram atendidos, às sextas-feiras, no turno da tarde, onde o paciente permanecia acompanhado apenas pelo pesquisador que lia as perguntas, sendo as respostas gravadas por um gravador da marca Philips, modelo DVT 4000, sem limite de tempo, com autorização prévia do entrevistado.

Os dados foram analisados com base na técnica de análise de conteúdo ${ }^{(5)}$, sendo posteriormente realizado seu reagrupamento em classes ou categorias. Dessa forma, abordam-se as seguintes etapas para categorização dos dados: pré-análise; leitura flutuante das entrevistas; constituição do corpus; seleção das unidades de contexto e de registro; recorte; codificação e classificação; categorização e definições das categorias simbólicas ${ }^{(5)}$.

A partir de então emergiram cinco categorias temáticas: Relação entre dor e cansaço; A fibromialgia resumida como uma dor pessoal, física e mental; Fisioterapia, a oficina funcional do corpo; A perspectiva do sadio e a incerteza; e $\mathrm{O}$ fisioterapeuta humanizado.

Esta pesquisa recebeu aprovação do Comitê de Ética em Pesquisa com Seres Humanos da Universidade de Fortaleza (COETICA - UNIFOR) sob o número 191/11, respeitou os aspectos éticos da Resolução 196/96 $6^{(6)}$ e obteve a assinatura do termo de consentimento livre e esclarecido de todos os investigados. Para manter o anonimato, os entrevistados foram chamados de pacientes (P). P1, P2, P3...

\section{RESULTADOS E DISCUSSÃO}

A seguir serão apresentados os dados de identificação dos sujeitos da pesquisa e, na sequência, os dados que emergiram das categorias temáticas.

\section{Dados de identificação dos sujeitos}

Realizaram-se 10 entrevistas com pacientes fibromiálgicos, sendo nove do sexo feminino e um do sexo masculino, apresentando idade entre 40 e 60 anos (média de 56 anos). Apenas um paciente trabalhava como ambulante, os demais executavam tarefas domésticas. Sobre a escolaridade, um paciente não era alfabetizado, oito tinham ensino fundamental e um ensino superior.

Um estudo epidemiológico com 500 mulheres com idade média de 50,16 anos revelou que no Brasil muitos pacientes com fibromialgia têm baixo nível educacional, cerca de 1/3 não haviam completado o ensino fundamental, e em relação à atividade ocupacional $31 \%$ estavam empregadas, $21 \%$ eram donas de casa e $34 \%$ encontravamse desempregadas ou aposentadas ${ }^{(7)}$. Estas informações estão em comum com os dados da presente pesquisa no que diz respeito à mediana das idades e à predominância de pacientes que não possuem atividades laborais.

A principal queixa apresentada pelos participantes do atual estudo foi a presença da dor constante, cujo sintoma será abordado a seguir, desde sua definição, repercussões físicas e emocionais, bem como suas implicações durante o tratamento.

\section{Relação entre dor e cansaço}

Na categoria "relação entre dor e cansaço", os pacientes se definiram como pessoas que se sentem cansadas e fadigadas devido à dor constante. Porém, aprenderam a superar a dor e a continuar suas vidas nas atividades funcionais. Como relatam:

"Hoje me defino como uma pessoa no estágio bem avançado da doença, cansada e chata por causa da dor. Quando a dor está ativada, acordada, ela me deixa com raiva, chateada, estressada, cansada. No dia a dia, eu evito ao máximo ficar desse jeito, mas a dor me deixa assim." (P1)

Em 1979, a Associação Internacional para o Estudo da Dor (IASP) cunhou a importante definição da dor como "uma experiência sensorial e emocional desagradável associada a dano real ou potencial de tecidos ou descrita em termos de tal dano". Porém, é conhecido o fato de que existe uma diferença entre a realidade objetiva de um estímulo doloroso e a resposta subjetiva a este. Quando se trata de dor crônica, existe uma diminuição do contacto social, levando-o a concentrar-se quase exclusivamente na 
dor. Esta tendência conduz a um círculo vicioso de dor, falta de atividade, medo, depressão e mais $\operatorname{dor}^{(8)}$.

"Com o problema que eu tenho, o que eu posso fazer eu faço, mesmo que eu não possa, eu vou tentar. Sei que aquilo está doendo muito, mas eu faço, mesmo que depois eu passe o dia deitada, porque tem muita gente pior que eu. A gente tem que aprender a saber viver e conviver com a doença." (P7)

A diminuição da capacidade funcional que foi relatada na presente pesquisa é evidenciada em outro estudo que mostra que a dor compromete a força e o desempenho para as atividades dos pacientes com fibromialgia, o que afeta diretamente a sua qualidade de vida. Além disso, os indivíduos que apresentam maiores dificuldades na realização das tarefas da vida diária percorrem menores distâncias quando avaliada sua capacidade funcional ${ }^{(9)}$.

A dor na fibromialgia é diferente de qualquer outra impressão sensorial. Suas manifestações dependem de aspectos sociais, psicológicos, culturais, entre outros, tornando sua expressão clínica altamente variada e, consequentemente, exigindo diferentes abordagens terapêuticas $^{(7)}$.

"Eu acho assim que tira um monte de coisa que você gostaria de fazer e não pode mas se é essa a alternativa [...] a cada dia que passa melhora, mas não sei se é porque eu já melhorei ou se me acostumei com a dor." (P4)

Em um estudo não foi possivel verificar uma relação direta da dor como estresse. No entanto, o comprometimento da funcionalidade nos pacientes com fibromialgia relacionase com uma maior percepção de estresse ${ }^{(10)}$.

Os sujeitos estudados no presente trabalho relatam que a dor transcende ao físico e não apresentam muitas perspectivas de melhora, devido ser uma patologia complexa e de difícil compreensão social, porém, procuram ajuda para superar suas dificuldades.

\section{A fibromialgia resumida como uma dor, pessoal, física e mental}

A categoria "a fibromialgia resumida como uma dor, pessoal, física e mental", remete ao conceito de que a síndrome da fibromialgia seja de diagnóstico eminentemente clínico, caracterizada por quadro de dor musculoesquelética crônica, associada a variados sintomas. Pode ser confundida com diversas outras doenças reumáticas e não reumáticas. Requer uma abordagem individualizada e multidisciplinar com a combinação de tratamento farmacológico e não farmacológico ${ }^{(11)}$.

Os pacientes do presente estudo definiram a fibromialgia como sendo uma dor crônica e sem cura, que afeta tanto o físico como a mente. Uma doença de difícil diagnose e acreditação, pois não aparece em exames. Cada um mostrou uma forma individual da percepção da dor. De acordo com os relatos:

"O que eu entendo é uma dor intensa, uma dor que a gente fica às vezes até comovida, porque eu já tentei tirar isso de mim, às vezes eu estava com tristeza, com angústia, mas eu não quero ficar assim pra não ficar com depressão, e, para isso, temos que saber viver, conviver! A dor é intensa, só sabe quem convive com ela!" (P7)

O diagnóstico da fibromialgia é essencialmente clínico, não havendo marcadores ou testes específicos para seu diagnóstico. Os exames auxiliares de diagnóstico servem para excluir outras doenças que podem se assemelhar ${ }^{(12)}$.

"É uma dor que pode dar no dedo, nas costas e não sai num raio- $x$, dói na perna e não sai na tomografia, não sai na ressonância a dor nas costas, porque é uma dor tão chata que parece ser uma dor no osso." (P10)

Entre 2010 e 2011 foram estipulados critérios pelo Colégio Americano de Reumatologia (ACR) para o diagnóstico da fibromialgia, levando em consideração outros sintomas, além da dor difusa, em detrimento da palpação dos pontos dolorosos. O paciente passa a preencher completamente o diagnóstico de fibromialgia caso apresente um índice de dor difusa $\geq 7 / 19$ e uma escala de gravidade $\geq 5$, ou índice de dor difusa entre 3-6 e escala de gravidade $\geq 9$. Os sintomas devem estar estáveis e presentes por pelo menos três meses, e não deve haver outra condição clínica que possa explicar essa sintomatologia ${ }^{(13)}$.

\section{Fisioterapia, a oficina funcional do corpo}

A categoria "a fisioterapia, a oficina funcional do corpo", informa que apesar dos pacientes terem consciência de que a fibromialgia não tem cura, eles relatam os bons resultados dos exercícios e alongamentos propostos pela fisioterapia. Principalmente a auto-percepção dos aspectos funcionais como caminhar, dormir e outros.

\begin{abstract}
"Quando eu esqueço tudo e vou fazer um pouco de alongamento, fico me sentindo bem melhor, até pra dormir e não tenho mais falta de sono, eu durmo bem, mas ainda acordo cansada, como se eu não descansasse. Pra mim, a fisioterapia é a melhor solução." (P2)
\end{abstract}

Alongamento é o termo usado para descrever os exercícios físicos que aumentam o comprimento das estruturas constituídas de tecidos moles e, consequentemente, a melhora da flexibilidade. Os efeitos do alongamento podem ser divididos em agudos e crônicos. Os agudos ou imediatos são resultado da flexibilização do componente elástico da 
unidade músculo tendínea. Já os efeitos crônicos resultam em remodelamento adaptativo da estrutura muscular, o que implica em aumento do comprimento muscular, que ajuda a suportar maior o estresse ${ }^{(14)}$.

Em um estudo, dentro das condições experimentais utilizadas com alongamentos e exercícios físicos, sugerese o tratamento fisioterápico, especialmente por meio de alongamentos musculares, gera um impacto positivo na fibromialgia, melhorando o sono e a rigidez das pacientes ${ }^{(15)}$.

“A fisioterapia pra mim foi essencial, se não existisse, eu estaria mil vezes pior. Acho que eu nem estaria andando direito porque o joelho dá muita dor, e principalmente debaixo do pé, que eu não conseguia nem caminhar direito, então depois que eu vim pra fisioterapia e com os tratamentos que o pessoal está fazendo, como o pilates, os exercícios de alongamento, eu hoje vejo que tem um leque de coisas diferentes e isso ajuda demais." (P3)

A fisioterapia visa diminuir os sintomas, melhorando o controle da dor e manutenção ou melhora das habilidades funcionais dos pacientes. Além disso, outra meta da fisioterapia deve ser o papel educativo, para que os ganhos da intervenção possam permanecer a longo prazo e os pacientes consigam se tornar menos dependentes dos cuidados de saúde. Incentivam-se estilos de vida mais participativos e funcionais que contribuam no restabelecimento físico e emocional do paciente ${ }^{(16)}$. Pela fala a seguir, vê-se concordância com o autor:

"Pelo tempo que eu estou fazendo, eu vejo que não é pra gente parar com a fisioterapia, porque se a gente parar é como um carro velho cheio de ferrugem, pega ele, sai de casa, ele vai, vai, vai, começa a engasgar, depois ele pega e falha e continua, dá uma acelerada e pronto... A fisioterapia é muito bom, inclusive o pilates, porque ele movimenta esse músculo aqui, esse daqui, esse aqui ó (paciente toca e aponta partes do corpo) então movimentando o músculo já vai melhorar alguma coisa." (P10)

As atividades físicas regulares contribuem não apenas para a melhora física, mas proporcionam benefícios psicológicos, melhoram e causam bem estar, além de eliminar a fobia aos exercícios. Os exercícios ativos induzem participação no enfrentamento de dor crônica. Os exercícios em grupo reduzem também os estresses psicológicos e facilitam a sua socialização ${ }^{(16)}$.

\section{A perspectiva do sadio e a incerteza}

A categoria "a perspectiva do sadio e a incerteza" traz a questão de que os pacientes com fibromialgia relataram ter uma esperança de ficar bons, ter uma vida normal e sadia com o avanço da medicina e pespectivas religiosas. Porém, ainda tiveram comentários sobre uma incerterza ou falta de precisão ao relatar sobre seu futuro.

"Quero sair boa daqui, poder sair correndo, voltar a correr, a caminhar, quero emagrecer! Indo a pé pra casa, fazer exercícios, ficar boa mesmo! E é isso que eu espero, que a fisioterapia me ajude a ficar boa, boa, boa mesmo. Sair daqui ótima e maravilhosa, arranjar um namorado." (P3)

"Eu não posso dar uma certeza de uns dias pra frente, porque eu tive melhoras e dessas melhoras eu me senti muito bem, mas, como tive uma recaida e agora eu estou na moderada e já fiquei na leve, na grave... Então, eu já passei pelos três periodos, as três etapas. Hoje eu estou moderada pra grave, então não dá pra ter uma escala de como amanhã eu vou estar." (P1)

A estratégia para o tratamento ideal da fibromialgia requer uma abordagem multidisciplinar com a combinação de modalidades de tratamentos não farmacológico e farmacológico. O tratamento deve ser elaborado, em discussão com o paciente, de acordo com a intensidade da sua dor, funcionalidade e suas características, sendo importante também levar em consideração suas questões biopsicossociais, e culturais. A dor crônica é um estado de saúde persistente que modifica a vida. O objetivo do seu tratamento é o seu controle e não sua eliminação ${ }^{(17)}$.

"Mudança de vida e comportamentos sempre é válido, os laços da família são de grande importância, eu fico pensando na minha família nas próximas gerações, dos que vão vir por aí, os filhos dos meus filhos, se eu vou estar fisicamente capaz de acompanhar o crescimento deles e curtir a infância deles." (P5)

Um estudo mostra que traumas desde a infância, mais principalmente fatores estressores, ansiedades e negligências com pacientes com fibromialgia estão diretamente relacionados com o estado do sistema muscular ${ }^{(18)}$,

"Bom, assim, a gente vai ficando velho e a tendência é os musculos e as juntas ficarem mais encriquilhados, mas como a gente continua fazendo os exercicios da fisioterapia, a tendência é ir melhorando, melhorando." (P10)

As intervenções físicas associadas a abordagens cognitivas comportamentais, tanto o toque como o exercício, produzem respostas motoras, autonômicas, neuroendócrinas, emocionais, comportamentais e de percepção corporal, reguladas pelo sistema límbico que organiza as expressões somáticas de estados emocionais e das experiências de pacientes com dor. Portanto, o fisioterapeuta deve ser um terapeuta educador para o paciente, independente da escolha por técnicas passivas 
ou ativas. Estratégias de educação para combater crenças disfuncionais, comportamentos anormais, pensamentos e atitudes negativas são capazes de modular a dor e aumentar a capacidade funcional dos pacientes ${ }^{(19)}$.

\section{O fisioterapeuta humanizado}

A categoria "o fisioterapeuta humanizado" trouxe relatos dos pacientes sobre o convívio com o fisioterapeuta, trazendo uma relação de afinidade e confiança. A preocupação do profissional em buscar a melhora de condição de doente do seu paciente e a sua postura como profissional são os predicados para realizar um tratamento com bons resultados.

"O que mais cura é a maneira como os profissionias nos tratam, a gente se sente amada. Eu acho que eles têm conhecimento sim, são bons demais. [...] Cada fisioterapeuta aqui faz um tratamento para cada paciente, tem um modo diferente para cada paciente e é de um jeitinho muito especial." (P2)

A Fisioterapia vem acompanhando a tendência de especialização e, com isso, vem se deparando com questões conflitantes. A construção do sujeito ético deve ser priorizada onde os profissionais lidam com seres humanos que vivenciam momentos de angústia e dor. O fisioterapeuta utiliza o toque como recurso terapêutico e é impossível tocar sem criar vínculo com o paciente. A prática do fisioterapeuta, além dos conhecimentos técnicocientíficos, exige maior envolvimento com o ser humano. É preciso empreender esforços no sentido de conciliar ética e ciência, pois nenhuma sociedade é capaz de pensar com sabedoria sem a comunicação desses dois saberes ${ }^{(20)}$.

"A pior coisa do mundo é parar no tempo e isso não acontece aqui. Vocês ficam sempre se renovando, parecem borboletas que saem dos casulos e vão procurar novos ares. São éticos, com uma postura ética, tratam a gente bem, a ética de vocês aqui é excelente, o tratamento é igual pra todo mundo, pode ser homem ou mulher, preto ou branco, é igual pra todo mundo." (P3)

$\mathrm{O}$ relacionamento de cuidado com o paciente requer uma atitude de sensibilidade. O paciente tem o direito de decidir sobre seu bem-estar e o fisioterapeuta precisa ser sensível aos sentimentos do paciente, respeitá-lo e não impor sua vontade ou ser paternalista. O fisioterapeuta precisa ser receptivo ao que o paciente requer em termos de reabilitação, para ser o sujeito do processo e não mais o objeto $^{(21)}$.

"A gente recebe muito amor e carinho e muita informação, orientação pra conviver com o problema da doença, a gente tem que saber viver e conviver com ela, a gente quando chega aqui já se sente outra pessoa, não é em casa emburricada e com dor que a gente vai melhorar. [...] Fisioterapeutas são ótimos, a gente não se sente com medo porque eu me acho nos braços e nas mãos de pessoas confiáveis, me sinto muito segura." (P7)

Os aspectos envolvidos no relacionamento entre aquele que trata e aquele que é tratado, indicando que a qualidade da relação que se estabelece entre os ambos é fundamental ao processo de adesão à fisioterapia. Um processo que deve ser fundamentado não apenas no número de sessões, mas na qualidade de cada uma delas ${ }^{(22)}$.

O fisioterapeuta tem como principal instrumento as mãos, que, através do toque, cuidam, reabilitam, confortam e curam. As mãos dos fisioterapeutas que operam modernos equipamentos também são aquelas que tocam e massageiam os usuários. O resgate do uso das mãos no contato direto entre profissional-doente está contribuindo para a humanização do atendimento e a valorização do toque, que vai de encontro à supervalorização das máquinas em detrimento de uma assistência humanizada ${ }^{(23)}$.

\section{CONSIDERAÇÕES FINAIS}

A maioria dos pacientes investigados se definiu como cansados e estressados devido à dor constante, sendo a principal reclamação e o fator casual da diminuição de suas atividades funcionais e laborais. Estando a dor diretamente relacionada com seu estado emocional, eles apresentaram a percepção de que precisam estar bem mentalmente para ter seu físico em melhores condições.

Apesar de saberem que a fibromialgia é uma enfermidade crônica e sem cura, apresentaram uma boa perspectiva em relação à melhora de sua condição física, porém, com a incerteza da manutenção dessa melhora. Compreendem também a importância e o papel das terapias físicas propostas pela fisioterapia, nesse processo de adaptação de seus limites físicos e da convivência com a dor.

Os pacientes têm o profissional fisioterapeuta como aliado permanente nesse processo e pela convivência, fazse um vínculo afetivo, que, regido pelos preceitos éticos torna o tratamento acolhedor, individualizado, confiante e satisfatório.

Os relatos mostraram que os pacientes, em atendimento fisioterapêutico, tem a percepção de sua condição como enfermo, sabendo da importância da fisioterapia na sua condição funcional. A presente narrativa dá um feedback ao profissional, identificando os objetivos e os fatores que interferem no tratamento, podendo sugerir também a necessidade de intervenções de outras especialidades. 


\section{REFERÊNCIAS}

1. Department of Health and Human Services (US), Centers for Disease Control and Prevention and National Institutes of Health. Arthritis types - overview: fibromyalgia. Atlanta; 2008 [acesso em 2014 Nov 14]. Disponível em: http:/www.cdc.gov/arthritis/basics/ fibromyalgia.htm

2. Häuser W, Bernardy K, Üçeyler N, Sommer C. Treatment of fibromyalgia syndrome with antidepressants: a metaanalysis. JAMA. 2009;301(2):198-208.

3. Brandão TO, Germano IMP. Experiência, memória e sofrimento em narrativas autobiográficas de mulheres. Psicol Soc. 2009;21(1):5-15.

4. Junges JR, Bagatini T. Construção de sentidos nas narrativas de doentes crônicos. Rev Assoc Med Bras. 2010;56(2):179-85.

5. Bardin L. Análise de conteúdo. $4^{\mathrm{a}}$ ed. Lisboa: Edições; 2008.

6. Brasil. Resolução CNS n 196 , de 10 de Outubro de 1996. Aprova diretrizes e normas regulamentadoras de pesquisa envolvendo seres humanos. Diário Oficial da União, 1996 Out 16; n. 201; Seção 1. p. 21082.

7. Rezende MC, Paiva ES, Helfenstein Jr. M, Ranzolin A, Martinez JE, Provenza JR, et al. EpiFibro - um banco de dados nacional sobre a síndrome da fibromialgia: análise inicial de 500 mulheres. Rev Bras Reumatol. 2013;53(5):382-87.

8. Kopf A, Patel NB. Guia para o Tratamento da dor em contextos de poucos recursos [Internet]. Washigton: Associação Internacional para o Estudo da Dor (IASP); [atualizado em 2010; acesso em 2013 Nov 3]. Disponível em: http://www.iasp-pain.org/AM/ Template.cfm?Section=Guide to Pain_Management in_Low_Resource_Settings\& Template $=/ \mathrm{CM} /$ ContentDisplay.cfm\&ContentID $=15927$

9. Homann D, Goes SM, Timossi LS, Leite N. Avaliação da capacidade funcional de mulheres com fibromialgia: métodos diretos e autorrelatados. Rev Bras Cineantropom Desempenho Hum. 2011;13(4):292-98.

10. Homann D, Stefanello JMF, Góes SM, Breda CA, Paiva ES, Leite N. Percepção de estresse e sintomas depressivos: funcionalidade e impacto na qualidade de vida em mulheres com fibromialgia. Rev Bras Reumatol. 2012;52(3):324-30

11. Helfenstein Júnior $\mathrm{M}$, Goldenfum MA, Siena CAF. Fibromialgia: Aspectos Clínicos e ocupacionais. Rev Assoc Med Bras. 2012;58(3):358-65.
12. Arnold LM, Clauw DJ, McCarberg BH. Improving the Recognition and Diagnosis of Fibromyalgia. Mayo Clin Proc. 2011;86(5):457-64.

13. Wolfe F, Clawn DJ, Fitzcharles MA, Goldemberg DL, Hauzer W, Katz RS, et al. Fibromyalgia criteria and severity scales for clinical and epidemiological studies: a modification of the ACR Preliminary diagnostic criteria for fibromyalgia. J Rheumatol. 2011;38(6):1113-22.

14. Di Alencar TAM, Matias KFS. Princípios fisiológicos do aquecimento e alongamento muscular na atividade esportiva. Rev Bras Med. 2010;16(3):230-4.

15. Bressan LR, Matsutani LA, Assumpção A, Marques AP, Cabral CMN. Efeitos do alongamento muscular e condicionamento físico no tratamento fisioterápico de pacientes com fibromialgia. Rev Bras Fisioter. 2008;12(2):88-93.

16. Batista JS, Borges AM, Wibelinger LM. Tratamento fisioterapêutico na síndrome da dor miofascial e fibromialgia. Rev Dor. 2012;13(2):170-4.

17. Heymann RE, Paiva ES, Helfenstein Junior M, Pollak DF, Martinez JE, Provenza JR et al. Consenso brasileiro do tratamento da fibromialgia. Rev Bras Reumatol. 2010;50(1):56-66.

18. Reis MJD, Rabelo LZ. Fibromialgia e estresse: explorando relações. Temas Psicol. 2010;18(2):399414.

19. Gosling AP. Mecanismos de ação e efeitos da fisioterapia no tratamento da dor. Rev Dor. 2012;13(1):65-70.

20. Machado D, Carvalho M, Machado B, Pacheco F. A formação ética do fisioterapeuta. Fisioter Mov. 2007;20(3):101-5.

21. Alves FD, Bigongiari A, Mochizuki L, Hossne WS, Almeida M. O preparo bioético na graduação de Fisioterapia. Fisioter Pesqui. 2008;15(2):49-56.

22. Subtil MML, Goes DC, Gomes TC, Souza ML. O relacionamento interpessoal e a adesão na fisioterapia. Fisioter Mov. 2011;24(4):745-53.

23. Silva ID, Silveira MFA. A humanização e a formação do profissional em fisioterapia. Ciênc Saúde Coletiva. 2011;16(Supl 1):1535-46.

\section{Endereço para correspondência:}

Fernanda Gadelha Severino

Av. Washington Soares, 1321

Bairro: Edson Queiroz

CEP: 60811-905 - Fortaleza - CE - Brasil.

E-mail: gadelhafernanda@yahoo.com.br 